\title{
Ovarian Dermoid Cyst with Secondary
}

\section{Sarcoma}

National Cancer Institute

\section{Source}

National Cancer Institute. Ovarian Dermoid Cyst with Secondary Sarcoma. NCI

Thesaurus. Code C40009.

A secondary sarcoma that has developed in an ovarian dermoid cyst. Representative examples of secondary sarcomas include leiomyosarcoma, fibrosarcoma, and angiosarcoma. 